Théologiques

Théologiques

\title{
Traduire la terminologie du bouddhisme à la lumière de la pratique du dharma
}

\section{Nicole Martínez-Melis}

Volume 15, numéro 2, 2007

La traduction des textes sacrés

URI : https://id.erudit.org/iderudit/017775ar

DOI : https://doi.org/10.7202/017775ar

Aller au sommaire du numéro

\section{Éditeur(s)}

Faculté de théologie et de sciences des religions, Université de Montréal

\section{ISSN}

1188-7109 (imprimé)

1492-1413 (numérique)

Découvrir la revue

\section{Citer cet article}

Martínez-Melis, N. (2007). Traduire la terminologie du bouddhisme à la lumière de la pratique du dharma. Théologiques, 15(2), 115-132.

https://doi.org/10.7202/017775ar
Résumé de l'article

L'implantation du bouddhisme en Occident comme tradition vivante conduit à reposer le problème de la traduction de sa terminologie sous un éclairage nouveau, ouvrant ainsi un champ d'étude qui apporte de nouvelles données à la traductologie. Le groupe de recherche Marpa du Departament de Traducció i d'Interpretació de l'Universitat Autònoma de Barcelona présente ici quelques résultats des travaux préliminaires en vue de l'élaboration d'une banque de données terminologique multilingue conçue comme aide à la traduction des textes du bouddhisme tibétain en espagnol et en catalan.
Tous droits réservés ( $\odot$ Faculté de théologie et de sciences des religions, Université de Montréal, 2007
Ce document est protégé par la loi sur le droit d'auteur. L’utilisation des services d'Érudit (y compris la reproduction) est assujettie à sa politique d'utilisation que vous pouvez consulter en ligne.

https://apropos.erudit.org/fr/usagers/politique-dutilisation/ 


\title{
Traduire la terminologie du bouddhisme à la lumière de la pratique du dharma
}

\author{
Nicole MARTÍNEZ-MELIS ${ }^{1}$ \\ Departament de Traducció i d'Interpretació, \\ Universitat Autònoma de Barcelona
}

L'intérêt croissant pour le bouddhisme au cours de ces dernières décennies a pour conséquence une augmentation notable du volume de traductions en langues occidentales de textes ayant trait à cette tradition spirituelle. Cet intérêt, comme l'avait déjà annoncé Henri Arvon (1996, 120) dès 1951, dépasse largement le domaine des études universitaires et se matérialise en une nouvelle implantation du bouddhisme loin de sa terre natale (Midal, 2006; Gira, 1989). En effet, à partir des années soixante, en Europe, en Australie et sur le continent américain, le bouddhisme n'est plus seulement un sujet réservé aux seuls érudits, mais devient aussi pour certains un chemin spirituel.

Cette nouvelle situation, qui pose le problème de la traduction au sens large et de la traduction de la terminologie en particulier, ouvre un champ d'étude nouveau qui rend une fois de plus manifeste le caractère interdisciplinaire de la traductologie. Le groupe de recherche MarpaTerm de l'Universitat Autònoma de Barcelona (Departament de Traducció i d'Interpretació) présente ici quelques résultats de ses travaux préliminaires, en vue de l'élaboration d'une banque de données terminologique, conçue comme aide à la traduction des textes du bouddhisme tibétain en espagnol et en catalan.

Nous expliquerons, tout d'abord, en quoi consiste le corpus que constituent les textes du bouddhisme tibétain, pour en indiquer la grande variété et nous délimiterons le champ de notre recherche. Nous présenterons ensuite une proposition de classification, dans la traduction des textes du

1. L'auteure rend ici compte de l'avancement des recherches du groupe MarpaTerm, qu'elle dirige, signant ainsi de façon collective. Ce groupe est composé des chercheurs et chercheuses suivants: M. Castellà, B. Llorca, N. Martínez-Melis, B. Mesa, M. Orozco, H. Roura, P. Sánchez-Gijón. 
bouddhisme tibétain, selon les catégories suivantes: contexte, finalité et récepteurs de la traduction, traducteurs, méthodes et types de traduction, langue à partir de laquelle on traduit et poids accordé à la langue originale réelle. Nous expliquerons aussi, à partir de concepts de l'anthropologie sociale et culturelle, en quoi consistent les présupposés théoriques qui servent de base à notre recherche terminologique. Pour finir, nous parlerons de l'élaboration de la banque de données et plus particulièrement des langues qui ont été retenues, ainsi que des techniques qui sont utilisées pour traduire la terminologie.

\section{Le corpus et le champ d'étude}

Le corpus que constitue la traduction des textes du bouddhisme tibétain est extrêmement vaste, dans la mesure où il est actuellement constitué de deux ensembles. D'une part, les textes indiens traduits du sanscrit en tibétain ${ }^{2}$ et les exégèses des maîtres et érudits tibétains au cours des siècles; d'autre part, les œuvres contemporaines qui sont généralement transcrites d'enseignements oraux donnés par de prestigieux maîtres de la diaspora tibétaine ${ }^{3}$. Ce deuxième ensemble de textes, qui pour la tradition bouddhiste tibétaine est essentiel, puisqu'elle est toujours transmise oralement de maître à disciples, est à son tour constitué de deux groupes. Il faut distinguer les textes originaux en tibétain, traduits pour la plupart en anglais, des textes originaux en anglais, dont les auteurs sont des maîtres exilés ou nés en exil, qui utilisent l'anglais pour transmettre leur sagesse à la communauté occidentale ${ }^{4}$.

On constatera que nous n'utilisons pas le terme de textes sacrés. Nous ne faisons pas de différence entre les textes attribués au bouddha historique et les commentaires des maîtres historiques, ni entre les textes classiques et les textes modernes. Cela pour deux raisons: l'une d'ordre pratique, l'autre d'ordre théorique. Premièrement, notre recherche portant sur la terminologie, cette différence n'a pas lieu d'être, puisque ce sont les mêmes concepts qui sont expliqués depuis toujours. Deuxièmement, d'un point de vue philosophique, nous nous demandons si qualifier de sacrés

2. Le Kanjur (bka' 'gyur), les enseignements du Bouddha, et le Tenjur (bstan 'gyur), les commentaires, représentent, dans l'édition de Narthang (snar thang), 325 volumes comprenant 4569 textes traduits du sanscrit, hormis quelques rares exceptions (Guillon 1995, 103).

3. Il faut signaler, en outre, les textes faisant partie des trésors spirituels (gter ma) qui sont des objets variés cachés par des êtres éveillés et découverts à l'époque appropriée (Dilgo Khyentsé 1996, 256).

4. Nous ne tenons pas compte ici des enseignements donnés par des disciples occidentaux. 
les textes canoniques, fondateurs ou anciens, ne serait pas une erreur, car cela impliquerait tout d'abord de se demander si la dichotomie sacré / profane peut s'appliquer au bouddhisme, de la même manière qu'elle est appliquée dans les religions monothéistes - qu'on appelle aussi religions du livre. C'est une question importante que nous n'aborderons pas ici, parce qu'elle nous engagerait dans une analyse d'autres oppositions, notamment entre philosophie et religion, foi et raison, lorsqu'elles sont appliquées à la tradition bouddhiste.

Pour délimiter notre champ d'étude, il nous faut tout d'abord examiner les deux domaines où le bouddhisme tibétain est étudié : l'étude dans le cadre académique et l'étude hors du cadre académique.

Dans le cadre académique, le bouddhisme est étudié dans des contextes variés, dont les objectifs diffèrent. Il s'agit souvent d'une matière ou d'un sujet d'étude dans des disciplines comme les langues et cultures orientales, les études religieuses, la philosophie, l'anthropologie et l'histoire de l'art. Dans de nombreux pays occidentaux, le bouddhisme est enseigné et fait l'objet de travaux de recherches dans des centres et des sections universitaires de langues et de civilisations orientales, d'études orientales ou asiatiques ${ }^{5}$, etc. C'est aussi un sujet d'enseignement en études religieuses, science des religions, études culturelles et historiques des religions, religion comparée, etc. Considéré comme une philosophie autant qu'une religion, le bouddhisme est, en outre, étudié dans les facultés ou départements de philosophie, dans le cadre de cours sur les philosophies de l'Inde ou les philosophies asiatiques. Finalement, il peut être étudié dans une perspective anthropologique, mais aussi d'un point de vue artistique, puisque l'art bouddhique figure souvent comme sujet d'étude dans l'histoire de l'art.

Hors du cadre académique, le bouddhisme est étudié, nous semble t-il, pour deux raisons différentes. La première est d'ordre intellectuel, elle correspond a une certaine curiosité, un désir de découvrir, de connaître d'autres courants de pensée, et ce désir d'enrichissement personnel ne vise qu'un savoir encyclopédique. La deuxième raison est d'ordre spirituel, elle correspond à un désir d'approfondir la connaissance d'une doctrine philosophique et religieuse à laquelle on adhère. Contrairement à la première, dans cette deuxième situation, il s'agit d'étudier une tradition vivante, de suivre Bouddha et, pour parcourir ce chemin de libération, les enseignements

5. Il n'existe pas de tradition d'études asiatiques dans le cadre de l'université en Espagne, celles-ci commencent tout juste à voir le jour. 
oraux sont les piliers sur lesquels repose l'étude. C'est dans ce contexte que s'inscrivent les travaux dont nous présentons quelques résultats.

\section{La traduction du bouddhisme tibétain}

L'étude du bouddhisme dans les différents contextes que nous venons d'évoquer pose toutes sortes de problèmes traductologiques dérivés de la diversité pour ce qui est de la finalité de la traduction et des attentes des récepteurs. Il nous a donc paru important d'élaborer une proposition de classification pour la traduction de textes bouddhiques dans les deux domaines d'étude (académique ou non), en tenant compte du contexte, de la finalité et des récepteurs de la traduction, des traducteurs, des méthodes, des modalités et des types de traduction ${ }^{6}$, de la langue à partir de laquelle la traduction est réalisée (langue orientale, classique ou moderne et langue occidentale) et du poids accordé à la langue originale réelle (langue orientale classique). Il s'agit d'une classification élaborée à partir de notre travail sur le bouddhisme tibétain, notre intention étant de faire une première ébauche d'un instrument qui facilite la réflexion sur cet objet de recherche et qui ne prétend pas couvrir toutes les traditions bouddhistes.

\subsection{La traduction dans le cadre académique}

On peut distinguer, dans le cadre académique, deux contextes de traduction du bouddhisme. D'une part, la traduction dans les études de langues orientales, d'autre part, la traduction dans les études de philosophie, de religion, d'anthropologie et d'art. Dans le premier contexte, la traduction a pour principal objectif d'enseigner, d'approfondir la connaissance d'une langue/culture dans laquelle le bouddhisme occupe une place privilégiée. Le récepteur est généralement un spécialiste du sujet (enseignant, chercheur) ou une personne en cours de spécialisation (étudiant); les auteurs des traductions étant des universitaires eux-mêmes et, à l'occasion, des étudiants de $3^{\text {e }}$ cycle. La langue originale de la traduction est la langue orientale classique; il s'agit d'une version et toujours d'une traduction écrite. Pour ce qui est de la méthode, ces traductions érudites et annotées suivent la méthode philologique qui implique l'introduction de commentaires variés (philologiques, historiques, doctrinaires, etc.) et sont le plus souvent accompagnées

6. Nous suivons ici les définitions données par Hurtado Albir (2001) pour méthode, modalité et types de traduction. 
du texte original — l'édition pouvant avoir été établie par le traducteur lui-même -, ainsi que de glossaires, parfois même d'index de mots ou de noms. Le poids accordé à la langue originale est élevé, à tel point que le traducteur inclut des parenthèses, soit pour indiquer qu'il a ajouté des éléments morphologiques et syntaxiques dans la langue d'arrivée, afin de faciliter la lisibilité du texte, soit pour donner en translitération académique des mots-clés dans leur langue originale.

La traduction dans le contexte des études de philosophie, de religion, d'anthropologie et d'art a pour principal objectif l'étude d'une religion et d'une philosophie afin de les connaître, de les comprendre et de les comparer à d'autres religions, d'autres philosophies. La langue originale orientale classique n'a pas autant de poids que dans le contexte précédent, même si certains enseignants et chercheurs connaissent et étudient cette langue. Les traductions utilisées ont généralement été réalisées par les universitaires spécialisés en langues orientales et, dans certains cas, par des spécialistes de philosophie ou de religion. Notons qu'ici, la langue originale peut souvent être, outre la langue orientale, une langue occidentale. C'est ce qu'il advient lorsque le texte original est, soit un texte en langue occidentale, soit une traduction occidentale d'un texte classique. En outre, si le poids accordé à la langue originale réelle est généralement moins élevé que dans le contexte universitaire précédent, le traitement de la terminologie est très semblable. La méthode de traduction balance entre la méthode philologique (voir plus haut) et la méthode littérale qui consiste à tenter de reconstruire les éléments linguistiques du texte original.

\subsection{La traduction hors du cadre académique}

Hors du cadre académique, on peut aussi distinguer deux contextes de traduction des textes bouddhiques. D'une part, la diffusion des philosophies et religions orientales dans l'édition commerciale ${ }^{7}$ d'autre part, la pratique de la doctrine bouddhiste, autrement dit, la pratique du dharma. Dans le premier contexte, les traductions sont très variées, depuis la traduction d'un texte canonique à partir de la langue originale réelle, jusqu'à la traduction pour une encyclopédie d'un article sur le bouddhisme rédigé dans une langue occidentale. Les traducteurs sont soit des universitaires, soit des traducteurs professionnels qui, très souvent, ne connaissent pas le sujet.

7. Nous ne parlons pas ici des maisons d'éditions spécialisées dans le bouddhisme et créées par des communautés bouddhistes. 
Le poids accordé à la langue originale réelle n'est pas très élevé, voire nul, selon les publications, les textes, la langue à partir de laquelle la traduction est réalisée et la finalité implicite ou explicite de la traduction. Quant à la méthode de traduction suivie, outre la méthode philologique et la méthode littérale, dont nous venons de parler, on constate dans ce contexte l'utilisation de la méthode interprétative-communicative, pour laquelle l'objectif principal est de maintenir la fonction et le genre de l'original, afin de produire le même effet chez le destinataire, ainsi que de la méthode libre, qui implique divers degrés d'éloignement par rapport au texte original, dans ses dimensions sémiotiques et/ou de communication.

Dans le contexte de la pratique du dharma, la traduction a pour finalité de transmettre une tradition spirituelle et devrait être une source d'inspiration. Les traductions sont réalisées à partir de textes originaux et de traductions, et les traducteurs sont tous bouddhistes, qu'ils soient universitaires (spécialistes ou non du sujet), traducteurs professionnels ou amateurs, laïques ou religieux, regroupés en comités de traduction. La langue originale est très souvent la langue orientale, classique dans le cas des textes classiques, moderne dans le cas des enseignements. Une des particularités de ce contexte de traduction est que la langue anglaise joue très souvent le rôle de langue originale. D'une part, l'anglais est véritablement la langue originale dans le cas des textes qui sont des transcriptions d'enseignements actuels donnés dans cette langue par des maîtres tibétains. D’autre part, les publications sur le bouddhisme tibétain en langue anglaise, qu'il s'agisse de traductions du tibétain ou de textes originaux, représentent un volume qui dépasse très largement n'importe qu'elle autre langue occidentale. L'anglais est donc véritablement une langue source, mais sert aussi d'intermédiaire entre le tibétain et les autres langues occidentales, notamment l'espagnol et le catalan. La méthode de traduction, comme dans le contexte de l'édition commerciale, oscille entre quatre méthodes: philologique, littérale, interprétative-communicative et libre. Pour ce qui est du poids accordé à la langue originale réelle, selon la situation de traduction, il peut être très élevé ou nul. On trouve finalement, dans ce contexte, deux aspects concernant les modalités et le type de traduction qui lui sont propres. Nous avons vu, par exemple, dans les autres contextes, que la modalité était toujours la traduction écrite, or, dans le cadre des enseignements, la traduction est orale : elle peut être simultanée, consécutive ou à vue; par ailleurs, la traduction écrite est parfois la traduction d'une transcription d'un enseignement oral. Il en va de même pour le type de traduction. Nous avons vu 
précédemment qu'il s'agissait presque toujours d'une version; pour les enseignements, en revanche, il peut parfois s'agir d'un thème, c'est à dire d'une traduction de la langue maternelle vers une langue étrangère, mais aussi d'une langue étrangère vers une autre langue étrangère.

\subsection{Tableau: la traduction du bouddhisme tibétain}

Nous présentons ci-dessous un résumé, sous forme de tableau, en ce qui concerne la traduction des textes du bouddhisme tibétain. Il s'agit là d'une simple ébauche qui, malgré le côté un peu artificiel des catégories, lesquelles en réalité se recoupent parfois, nous permet d'apprécier toute la richesse de l'approche traductologique dans ce champ de recherche qu'est la traduction dans la pratique de la doctrine bouddhiste et qui, hormis quelques exceptions (Doboom Tulku 1995; Bédard 1995; Martínez-Melis 2005; Foiera 2005 ; Crosby 2005; Grupo Marpa 2006), est très peu étudié sous cet angle.

\section{LA TRADUCTION DU BOUDDHISME TIBÉTAIN}

\begin{tabular}{|c|c|c|c|c|}
\hline & \multicolumn{2}{|c|}{ cadre académique } & \multicolumn{2}{|c|}{ hors du cadre académique } \\
\hline $\begin{array}{l}\text { contexte } \\
\text { de traduction }\end{array}$ & $\begin{array}{c}\text { langues } \\
\text { orientales }\end{array}$ & $\begin{array}{c}\text { philosophie, } \\
\text { religion, } \\
\text { anthropologie, } \\
\text { art }\end{array}$ & $\begin{array}{l}\text { édition } \\
\text { commerciale } \\
\text { religion, } \\
\text { philosophie }\end{array}$ & $\begin{array}{l}\text { pratique du } \\
\text { dharma }\end{array}$ \\
\hline $\begin{array}{l}\text { finalité } \\
\text { de la traduction }\end{array}$ & $\begin{array}{c}\text { étudier une } \\
\text { langue/ } \\
\text { culture }\end{array}$ & $\begin{array}{l}\text { connaître, } \\
\text { comprendre, } \\
\text { comparer }\end{array}$ & $\begin{array}{l}\text { divulguer } \\
\text { des aspects } \\
\text { culturels }\end{array}$ & $\begin{array}{l}\text { transmettre une } \\
\text { tradition } \\
\text { spitituelle, } \\
\text { être une source } \\
\text { d'inspiration }\end{array}$ \\
\hline $\begin{array}{l}\text { récepteurs } \\
\text { de la traduction }\end{array}$ & spécialistes & spécialistes & tout public & practiquants \\
\hline traducteurs & universitaires & universitaires & $\begin{array}{l}\text { universitaires, } \\
\text { professionnels }\end{array}$ & $\begin{array}{l}\text { universitaires, } \\
\text { amateurs, } \\
\text { comités de } \\
\text { traduction }\end{array}$ \\
\hline $\begin{array}{l}\text { méthodes } \\
\text { de traduction }\end{array}$ & philologique & $\begin{array}{c}\text { philologique, } \\
\text { littérale }\end{array}$ & $\begin{array}{c}\text { philologique, } \\
\text { littérale, } \\
\text { interprétative- } \\
\text { communicative, } \\
\text { libre }\end{array}$ & $\begin{array}{c}\text { philologique, } \\
\text { littérale, } \\
\text { interprétative- } \\
\text { communicative, } \\
\text { libre }\end{array}$ \\
\hline
\end{tabular}




\section{LA TRADUCTION DU BOUDDHISME TIBÉTAIN (suite)}

\begin{tabular}{l|c|c|c|c}
\hline & \multicolumn{2}{|c|}{ cadre académique } & \multicolumn{2}{c}{ hors du cadre académique } \\
\hline $\begin{array}{l}\text { modalités de } \\
\text { traduction }\end{array}$ & écrite & écrite & écrite & $\begin{array}{c}\text { écrite, orale } \\
\text { (simultanée, } \\
\text { consécutive, } \\
\text { à vue) }\end{array}$ \\
\hline $\begin{array}{l}\text { types } \\
\text { de traduction }\end{array}$ & version & version & version & $\begin{array}{c}\text { version, } \\
\text { thème }\end{array}$ \\
\hline $\begin{array}{l}\text { langue à partir } \\
\text { de laquelle on } \\
\text { traduit }\end{array}$ & $\begin{array}{l}\text { orientale } \\
\text { classique }\end{array}$ & $\begin{array}{c}\text { orientale } \\
\text { classique, } \\
\text { occidentale }\end{array}$ & $\begin{array}{c}\text { orientale } \\
\text { moderne, } \\
\text { occidentale }\end{array}$ & $\begin{array}{c}\text { orientale } \\
\text { classique, } \\
\text { orientale } \\
\text { moderne, } \\
\text { occidentale }\end{array}$ \\
\hline $\begin{array}{l}\text { poids accordé } \\
\text { à la LO réelle }\end{array}$ & très élevé & relatif & relatif, & $\begin{array}{c}\text { très élevé, } \\
\text { relatif, nul }\end{array}$ \\
\hline
\end{tabular}

Les différents domaines d'étude du bouddhisme ont ainsi produit et continuent à produire diverses méthodes de traduction qui font coexister plusieurs équivalents terminologiques pour un même concept. En outre, la situation peut se compliquer à l'extrême, lorsque ces équivalents sont le résultat d'une traduction à partir d'une langue relais, généralement l'anglais. Voyons par exemple le substantif tibétain sems can (prononcer "sem tchen») qui signifie "[être] doué de conscience", c'est à dire un être sensible au bienêtre et à la douleur, ce qui renvoie à un concept plus large que celui d' " être humain" et dont la traduction anglaise traditionnelle est sentient beings, l'emploi étant généralement au pluriel. Ce terme a été traduit par un calque en espagnol, seres sintientes, qui n'est pas très éclairant, même si l'adjectif sintiente, qui n'existe pas, peut être compris comme un dérivé du verbe espagnol sentir. Face à ce terme étrange, obscur, certains le traduisent plus récemment par seres sentientes, dont l'adjectif sentiente a pour avantage d'être consigné dans le Diccionario del español actual (Seco 1999), mais qui est malheureusement un terme rare, créé par le philosophe espagnol Xavier Zubiri et qui renvoie ainsi à un courant de pensée qui n'a rien à voir avec le bouddhisme. On trouve aussi ser vivo comme équivalent, signifiant «être vivant ». Toutefois, une distinction est généralement faite dans le bouddhisme, entre ce qui a une conscience et ce qui n'en a pas, plutôt qu'entre ce qui est vivant et ce qui ne l'est pas. D'autres le traduisent par seres animados, ce qui n'est pas non plus satisfaisant, car l'adjectif animado renvoie immédiatement 
au concept d'âme, ce qui pose également un problème, puisque le bouddhisme n'envisage pas l'âme telle qu'elle est conçue dans notre culture. Une dernière option de traduction est seres, c'est à dire "êtres » tout court. Comme nous venons de le constater, une trop grande variété d'équivalents, qui plus est, souvent inexacts, n'est pas souhaitable pour la transmission vivante du bouddhisme.

\section{Les présupposés théoriques de la recherche}

L'objectif principal de notre travail est d'instaurer une terminologie qui facilite la transmission et la compréhension du bouddhisme de tradition tibétaine en espagnol et en catalan en suivant des critères provenant de la terminologie et de la traductologie. Nous partons du constat selon lequel le bouddhisme a influencé de nombreuses cultures tout au long de son histoire et que son expansion, donc sa traduction, n'a pas été un obstacle à la continuité de valeurs qui ont été préservées, malgré la diversité culturelle avec laquelle il est entré en contact (Aguilar 1997). C'est pourquoi nous considérons que la meilleure manière de faciliter la transmission et la compréhension du bouddhisme en Occident est, d'une part, de tenir compte de sa capacité d'adaptation et, d'autre part, en appliquant à la traduction de la terminologie des concepts de l'anthropologie sociale et culturelle, d'éviter tout ethnocentrisme ou multiculturalisme pour miser sur une approche interculturelle, comme nous l'expliquons à présent.

\subsection{Ethnocentrisme et multiculturalisme}

L'ethnocentrisme est la tendance à interpréter le monde et les autres cultures à partir d'un point de vue unique, formé par les idées et les jugements de valeur d'un observateur (Beltrán 2005). Cette attitude pleine de préjugés, qui implique une survalorisation de notre propre culture - considérée supérieure aux autres - et donc une négation de l'autre, ne permet pas la rencontre. Dans la traduction de la terminologie, la tendance ethnocentriste se manifeste par ce que nous appelons une option méthodologique d'assimilation ${ }^{8}$, qui consiste à s'approprier un concept original et à effacer sa particularité en lui appliquant un terme de la langue d'arrivée, qui renvoie à un concept différent. On trouve des exemples de cette option, qui est caractéristique des traductions les plus anciennes, dans le Dictionnaire thibétain - latin - français de la mission catholique française du Tibet, réalisé

8. Cette option méthodologique correspond au domesticating de Venuti (1995). 
au XIX siècle. Les missionnaires français traduisent sdig pa qui signifie «ce qui dégrade " par "peccatum, culpa, vitium; péché, faute, vice", sans tenir compte du système philosophique et religieux auquel ce terme appartient. L'ethnocentrisme est de même une caractéristique de la première traduction occidentale du célèbre Livre des morts tibétain, datant de 1927, dans laquelle Evans-Wentz utilise une terminologie propre au christianisme et aux écrits de la Société théosophique (Prats 1999) qui, ainsi que d'autres mouvements ésotériques de la fin du XIX ${ }^{\mathrm{e}}$ siècle, tentèrent de s'approprier le bouddhisme.

Quant au multiculturalisme, nous l'employons ici dans le sens de reconnaissance, du fait de la diversité culturelle associée à une certaine tendance à maintenir les cultures séparées (Beltrán 2005). En d'autres termes, les différences sont reconnues mais restent bien délimitées, de sorte qu'il n'y a pas, là non plus, de rencontre possible. Dans la traduction, le multiculturalisme se manifeste par ce que nous appelons une option méthodologique de différenciation ${ }^{9}$, qui consiste à abuser de l'emprunt, tout en conservant la graphie originale, et du calque, lorsque cela n'est pas indispensable. Le traducteur, par exemple, ne traduit pas le terme alors qu'un équivalent existe dans la langue d'arrivée, ou bien, s'il n'y a pas d'équivalent, il procède à une traduction littérale sans tenir compte des contraintes de la langue d'arrivée. Ce procédé qui accentue l'exotisme, généralement employé dans les travaux académiques, ne facilite pas la compréhension des concepts originaux: il les maintient dans une autre culture, comme si tout rapprochement était impossible. Voyons trois exemples d'emprunts du sanscrit, largement répandus chez les spécialistes, que nous avons tirés d'une thèse publiée (Pezzali 1968). À certaines occasions ${ }^{10}$, le terme bodhi n'est pas traduit et l'auteure lui applique le genre original féminin. Elle parle en effet de «la bodhi", alors que le masculin conviendrait mieux au français, car il s'agit de l'Éveil. Le terme śūnyatā n'est pas non plus traduit ( «la manière d'être des choses (tathatā) est la sūnyatā », p. 119), alors que le correspondant en français est "vacuité", ni le terme dharma ( «La perfection du savoir est l'essence de tous les dharma ", p. 147) qui, dans ce contexte, peut être traduit par "phénomènes », et à qui l'auteure n'applique pas la marque du pluriel.

9. L'option méthodologique de différenciation et ce que Venutti (1995) appelle foreignizing ont en commun l'accent mis sur les différences. Il est à noter cependant que notre réflexion aboutit, avec la troisième option méthodologique que nous proposons, à un dépassement de la dichotomie traditionnelle que cet auteur reprend.

10. Par exemple: «il porte son attention ferme sur la bodhi », p. 69; «il accomplit le chemin gnoséologique pour parvenir à la bodhi », p. 127. 
Dans ce dernier exemple, le fait d'opter pour un emprunt — qui n'a pas lieu d'être puisqu'il existe un équivalent dans la langue d'arrivée - et de ne pas appliquer le pluriel ne peut que créer une confusion, dans la mesure où le terme dharma a plusieurs significations dans le contexte du bouddhisme ${ }^{11}$.

\subsection{Interculturalité et transculturalité}

L'interculturalité se caractérise par l'échange et la communication entre les cultures; il y a rencontre, sans hiérarchie ni désir de domination. Il s'agit d'une dynamique qui permet l'émergence de la transculturalité. L'objectif de l'approche transculturelle est de dépasser le culturel pour chercher un équilibre à travers une compréhension universelle, arriver en quelque sorte à créer des faits culturels nouveaux (Mancini 1999). Dans la traduction de la terminologie, cette approche se matérialise par ce que nous appelons l'option méthodologique de transculturisation ${ }^{12}$, c'est à dire, un va-et-vient raisonné entre les concepts et les termes des deux langues/cultures en contact, qui permet de décider, pour chaque situation, quelle technique de traduction, de l'équivalence à l'emprunt, est la plus appropriée. Il s'agit de trouver un équilibre pour communiquer un message dans ce qu'il a d'essentiel, pour créer quelque chose de nouveau qui est, en ce qui nous concerne, le bouddhisme dans deux langues latines: l'espagnol et le catalan.

Ainsi, ni l'approche ethnocentriste, pratiquée surtout par les premiers traducteurs - qui ne connaissaient peut-être pas suffisamment le sujet -, ni l'approche multiculturelle de la recherche académique traditionnelle - qui se cantonne le plus souvent dans la connaissance livresque - ne peuvent servir à traduire le bouddhisme en tant que tradition spirituelle vivante. Seule une approche interculturelle peut en relever le défi et intégrer la terminologie dans la langue/culture du récepteur.

\section{La banque de données terminologique}

Conçue comme une aide à la traduction, la banque de données de MarpaTerm a pour mission d'amorcer un processus de standardisation, en

11. Quant à nous, nous proposons de réserver l'emprunt «dharma » à la doctrine bouddhiste et de traduire les autres significations selon le contexte.

12. Il est a noté qu'avec cette troisième option méthodologique notre réflexion aboutit à un dépassement de la dichotomie traditionnelle en traduction, qui est soit de réduire les aspects culturels originaux à la culture du lecteur, soit de transporter ce lecteur dans la culture de départ. 
espagnol et en catalan, de la terminologie bouddhiste, notamment de tradition tibétaine. Cette standardisation nous apparaît indispensable pour parvenir à une bonne compréhension du bouddhisme et permettre une communication efficace, qui facilite la préservation de l'authenticité du bouddhisme dans la culture d'arrivée, ainsi que le signale Brownlie (2002, 308) à propos de la traduction des termes philosophiques.

\subsection{Les langues de la banque de données}

Il s'agit d'une banque de données qui a pour langue de départ le tibétain et pour langues d'arrivées l'espagnol et le catalan, aucun dictionnaire bilingue ne couvrant ces combinaisons linguistiques. Notre travail est prescriptif, dans la mesure où notre objectif est de proposer une terminologie raisonnée du bouddhisme tibétain en espagnol et en catalan. Il est aussi descriptif pour ce qui est du français et de l'anglais, qui sont les deux langues occidentales à partir desquelles les traductions en espagnol et en catalan sont réalisées, les traductions directes du tibétain étant encore rarissimes. Chaque fiche correspond à un concept et comprend le(s) terme(s) en tibétain - en alphabet tibétain et en translittération Wylie ${ }^{13}$ - accompagné d'une transcription phonétique simplifiée, le terme en espagnol accompagné d'une définition, le terme en catalan, en français, en anglais et en sanscrit. Le sanscrit ${ }^{14}$ a été inclus pour trois raisons. En premier lieu, la plus grande partie de la terminologie tibétaine provient d'une traduction du sanscrit. En second lieu, les termes du bouddhisme les plus répandus en Occident sont des emprunts du sanscrit. Finalement, le sanscrit permet parfois de trouver une solution de traduction, dans la mesure où le concept n'est pas toujours exprimé de la même manière qu'en tibétain.

\subsection{Les techniques de traduction utilisées}

Chaque fiche, en outre, donne une information sur la technique de traduction utilisée, afin de permettre au traducteur qui consulte la banque de données de mieux comprendre le processus de traduction suivi et d'opter, si cela est nécessaire, pour une autre technique qui conviendrait mieux à son contexte de traduction, ainsi qu'à la méthode de traduction qu'il a choisie.

13. Translitération du tibétain la plus fréquente qui a été proposée par Turrell Wylie en 1959.

14. En transcription latine fixée en 1894 par le Congrès des orientalistes. 
Nous présentons ci-dessous les différentes techniques de traduction ${ }^{15}$ qui sont utilisées et appliquées en suivant les fondements théoriques présentés auparavant.

Lorsque le concept existe dans la langue/culture d'arrivée, nous proposons, par ordre de priorité, les techniques suivantes:

a) l'équivalent: il s'agit d'une traduction qui inclut la totalité du concept original. Par exemple, transitoriedad (substantif de transitoire), pour traduire mi rtag pa (impermanence). Le terme impermanencia ${ }^{16}$ n'étant pas consigné dans les dictionnaires, il nous semble plus cohérent d'utiliser le terme transitoriedad qui existe dans la langue d'arrivée, dans la mesure où il renvoie à la totalité du concept bouddhiste, plutôt que d'opter pour un néologisme, même si du point de vue lexical il est tout à fait possible de créer impermanencia en faisant précéder d'un préfixe le terme permanencia qui, lui, figure dans les dictionnaires.

b) l'équivalent contextuel: il s'agit d'un terme de la langue d'arrivée qui renvoie à un concept ne couvrant pas la totalité du concept bouddhiste évoqué par le terme tibétain. Cette technique correspond à un enrichissement de la langue/culture d'arrivée, ce qui arrive fréquemment dans la traduction philosophique. En effet, le «terme philosophique est souvent un mot existant auquel le philosophe donne un nouveau sens ou un sens plus spécifique» (Brownlie 2002, 296). Par exemple, mente (esprit), conciencia (conscience), vacuidad (vacuité), sufrimiento (souffrance).

Lorsque le concept n'existe pas dans la langue/culture d'arrivée, nous proposons, par ordre de priorité, les techniques suivantes:

a) la création: il s'agit de créer un terme nouveau pour la langue/culture d'arrivée. C'est une technique qui est très utile, car elle permet souvent de parvenir plus facilement qu'avec le calque ou l'emprunt à la compréhension du nouveau concept. Le bouddhisme considère, par exemple, trois types de souffrance. Le premier type de souffrance correspond à ce que nous entendons d'ordinaire par souffrance, c'est à dire, toutes sortes de douleur physique ou mentale. Le deuxième est la souffrance engendrée par la nature transitoire des phénomènes. Dans le troisième type, il s'agit de la souffrance qui caractérise toute forme d'existence conditionnée. Nous avons opté pour traduire le premier type correspondant à la souffrance ordinaire

15. Notre proposition s'inspire de la classification des équivalents juridiques de Santamaria $(2006,218)$.

16. Impermanencia, qui est la traduction la plus courante en espagnol, est en fait un calque de l'anglais. 
et pour bien le distinguer des deux autres, par sufrimiento por el dolor (souffrance due à la douleur) au lieu d'opter pour le calque traditionnel sufrimiento del sufrimiento (souffrance de la souffrance) ${ }^{17}$ qui nous semble peu éclairant, non seulement à cause de la répétition du mot souffrance, mais aussi à cause de la construction grammaticale qui, en espagnol, pourrait laisser à penser que la souffrance souffre .

b) la périphrase: il s'agit d'expliquer le concept qui correspond à un seul terme dans la langue de départ, en utilisant plusieurs mots: une proposition, une expression, sachant qu'un seul terme ne peut rendre le concept dans la langue d'arrivée. Par exemple, pour traduire «le Bouddha » el que ha despertado (celui qui s'est éveillé, qu'on traduit en français par «l'Éveillé »).

c) le calque: il s'agit d'une traduction littérale, lexicale, du terme tibétain ou sanscrit. Par exemple, el Despertar (l'Éveil), sufrimiento por el cambio (souffrance due au changement). Il est important ici d'insister sur la différence entre le calque et l'équivalent contextuel. Par exemple, le terme "souffrance " est un équivalent contextuel car, même s'il le dépasse, il renvoie parfois au concept de souffrance tel que nous le comprenons généralement. Par contre, "souffrance due au changement " est un concept complètement nouveau. Finalement, pour ce qui est du calque, un de nos objectifs est de réviser les calques de l'anglais erronés qui sont utilisés en espagnol (voir supra seres sintientes).

d) l'emprunt: il s'agit ici de ne pas traduire mais d'adapter le terme tibétain ou sanscrit au système de la langue d'arrivée. Ainsi buddha devient "buda» pour s'adapter aux normes de la langue espagnole; il est par ailleurs consigné dans les dictionnaires. Bodhisattva devient "bodisatva". Nous suivons à ce sujet Martínez de Sousa ${ }^{18}$ (2001, 129), pour qui l'emprunt peut être intégré, c'est à dire complètement adapté au système phonologique et graphique, ou bien acclimaté, dans les cas où il est consacré par l'usage sous une forme qui n'est pas complètement adaptée au système phonologique et graphique, (par exemple, dharma). Ainsi, nous avons décidé d'accepter les emprunts intégrés et acclimatés qui sont consacrés par l'usage, et d'opter dorénavant pour l'intégration des nouveaux emprunts quand ils seront nécessaires.

17. En sanscrit: duhkha duhkhata; en tibétain: sdug bsngal gyi sdug bsngal.

18. Martinez de Sousa est un grand spécialiste de la langue espagnole, notamment en orthographe, en typographie et en lexicographie. 


\section{Conclusion}

Nos travaux préliminaires à l'élaboration de la banque de données terminologique du bouddhisme tibétain mettent en lumière quatre contextes de traduction, parmi lesquels celui de la pratique du dharma, qui réunit à lui seul toutes les catégories traductologiques servant de base à la réflexion. Ils aboutissent également 1) à une option méthodologique de traduction qui permet d'aller au-delà de la dichotomie, privilégiant soit la langue/culture d'arrivée, soit la langue/culture de départ, 2) à l'élaboration de critères et de techniques de traduction devant permettre une véritable intégration de la terminologie.

La particularité de notre recherche réside, en outre, dans le fait que, ne trouvant pas satisfaisantes les nombreuses propositions des glossaires disponibles et réalisés à partir de l'anglais, nous ne partons pas seulement du terme dans la langue originale, mais surtout du concept. C'est la définition qui, situant le terme dans son contexte, nous guide. Nous sommes conscients que nous travaillons sur un matériau étranger à nos catégories de pensée. Nous devons d'abord le reconnaître, afin de nous en dessaisir et être entièrement à l'écoute de catégories nouvelles. Comme Wallace (2003, 5) l'a écrit à propos du bouddhisme et de la science ${ }^{19}$ :

To understand Buddhism on its own terms, it is imperative that we in the West recognize the cultural specificity of our own terms religion, philosophy, and science and not assume from the outset that Buddhism will somehow naturally conform to our linguistic categories and ideological assumptions.

19. Cité par Payne, 2002: 2. «Pour que nous puissions, en Occident, comprendre le bouddhisme dans sa propre terminologie, il est indispensable que nous reconnaissions la spécificité culturelle de nos termes de religion, philosophie et science, et que nous ne supposions pas dès le départ que le bouddhisme s'adapte tout naturellement à nos catégories linguistiques et conceptuelles ". 


\section{Références}

Aguilar, O. (1997), «La expresión de lo Absoluto en las diferentes culturas budistas: literatura o experiencia ?", M. Barcardí, dir., Actes del II Congrés Internacional sobre Traducció, Bellaterra, Universitat Autònoma de Barcelona.

Arvon, H. $\left(1996^{17}\right)$ [1951], Le bouddhisme, Paris, Presses Universitaires de France (Que sais-je?).

BÉDARD, S. (1995), "Cette sagesse qui bouscule ", Meta, 40/4, p. 564-578.

BELTRÁN, J. (2005), La interculturalitat, Barcelona, UOC.

BRownLIE, S. (2002), "La traduction de la terminologie philosophique", Meta, 47/3, p. 295-309.

Crosby, K. (2005), "What Does Not Get Translated in Buddhist Studies and the Impact on Teaching ", dans L. Long, dir., Translation and Religion: Holy Untranslatable?, Clevedon, Multilingual Matters, p. 4153.

Dictionnaire thibétain-latin-français (1986) [1899], Montignac, Association des Hautes Études en Tibétologie.

Dilgo Khyentsé, (1996), Le trésor du cour des êtres éveillés, trad. par le Comité de traduction Padmakara, Paris, Seuil (Points Sagesse).

Dовоом тulku, dir., (1995), Buddhist Translation. Problems and Perspectives, New Delhi, Manohar.

Evans-WenTZ, W.Y. (1954) [1927], The Tibetan Book of the Dead, Oxford University Press.

FolerA, M. (2005), «When East meets West via Translation: The Language of Soka Gakkai in Italy ", dans L. Long, dir., Translation and Religion: Holy Untranslatable, Clevedon, Multilingual Matters, p. 173-187.

GIRA, D. (1989), Comprendre le bouddhisme, Paris, Bayard/Centurion.

GRUPO MARPA (2006), "Traduction, sens et vérité, dans la tradition bouddhiste tibétaine ", Le sens en traduction, Paris, Minard (Lettres Modernes).

Guillon, E. (1995), Les philosophies bouddhistes, Paris, Presses Universitaires de France (Que sais-je?).

Hurtado Albir, A. (2001), Traducción y traductología. Introducción a la traductología, Madrid, Cátedra. 
ManCINI, U. (1999), "Multiculturalidad, Interculturalidad, Transculturalidad: Conceptos y Estrategias ", $1^{\circ}$ Congreso Internacional de Cultura y Desarrollo (La Habana), <http://www.crim.unam.mx/Cultura/ponencias/1CultDesa/CDIDE15.htm>

Martínez de Sousa, J. (2001²), [2000], Manual de estilo de la lengua española, Barcelona, Trea.

MARTínEZ-MELIS, N. (2005), «The Bodhicaryāvatāra translated into western languages ", dans A. Branchadell et al., dir., Less Translated Languages, Amsterdam-Philadelphia, John Benjamins, p. 207-224.

Midal, F. (2006), Quel bouddhisme pour l'Occident? Paris, Seuil.

PAYNE, R.K. (2002), «Buddhism and Cognitive Science: Contributions to an Enlarged Discourse », The Pacific World Journal, 3/4, p. 1-13.

PezZali, A. (1968), Śāntideva. Mystique bouddhiste des VII et VIII e siècles, Firenze, Vallechi.

PRATS, R. trad. (1996), El libro de los muertos tibetano: la liberación por audición durante el estado intermedio, Madrid, Siruela.

Rahula, W. (1961), L'enseignement du Bouddha, Paris, Seuil.

SANTAMARIA, L. (2006), "Els procediments de traducció en els textos jurídics », dans E. Monzó, dir. Les plomes de la justícia. La traducció al catalá dels textos jurídics, Barcelona, Pórtic (Biblioteca Universitària), p. 209-220.

Seco m., Andrés o., Ramos G. (1999), Diccionario del español actual, Madrid, Aguilar.

Wallace, B. A. (2003), "Introduction: Buddhism and Science", dans B.A. Wallace, dir., Buddhism and Science: Breaking New Ground, New York, Columbia University Press, p. 9-10. 


\section{Résumé}

L'implantation du bouddhisme en Occident comme tradition vivante conduit à reposer le problème de la traduction de sa terminologie sous un éclairage nouveau, ouvrant ainsi un champ d'étude qui apporte de nouvelles données à la traductologie. Le groupe de recherche Marpa du Departament de Traducció i d'Interpretació de l'Universitat Autònoma de Barcelona présente ici quelques résultats des travaux préliminaires en vue de l'élaboration d'une banque de données terminologique multilingue conçue comme aide à la traduction des textes du bouddhisme tibétain en espagnol et en catalan.

\section{Abstract}

The translation of Buddhist terminology has had to be rethought in the light of the practice of Buddhism in the West as a living tradition. This new area of research has already made a contribution to translation studies. In this article, the Marpa research group in the Departament de Traducció i d'Interpretació de l'Universitat Autònoma de Barcelona presents the results of some preliminary studies aimed at building a multilingual terminology data bank designed as an aid to translating Tibetan Buddhist texts into Spanish and Catalan. 